\title{
Ruptura del músculo orbicular de los labios en un músico de viento (síndrome de Satchmo), a propósito de un caso
}

\author{
Rupture of the orbicular oris muscle in a wind instrument player (SATCHMO \\ Syndrome). About a particular case
}

\section{F. Maneiro}

INSS Cantabria. España.

\section{Recibido: 21-10-14}

Aceptado: 03-11-14

\section{Correspondencia}

Francisco Maneiro Higuera

Calle Luis Hoyos Sainz n. $.^{\circ} 4 .^{\circ}$

39001 Santander. España.

Correo electrónico: francisco.maneiro@seg-social.es

Resumen

Los músicos profesionales son, además de artistas, trabajadores por cuenta ajena con patologías muy concretas relacionadas con su profesión. En ocasiones son tan específicas que no son bien conocidas por los clínicos generales o especialistas. Se presenta un caso de una patología casi exclusiva de los músicos que tocan instrumentos de viento de metal, que es la rotura del músculo orbicular de los labios que puede limitar o incluso impedir el continuar practicando de manera eficaz o completa la profesión. Se hace una revisión de la bibliografía y una descripción del síndrome, así como un análisis de los diferentes aspectos de la valoración de la posible incapacidad: menoscabos producidos, evaluación del trabajo, juicio clínico laboral y valoración de la contingencia.

Med Segur Trab (Internet) 2014; 60 (237) 779-785

Palabras clave: Orbicular, músico, Satchmo.

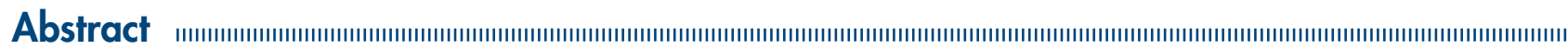

Professional musicians are not only artists but employed workers with very specific diseases related to their professions. Such diseases are sometimes so specific that they are not well known by general practitioners or specialists. We deal with an almost exclusive pathology of musicians playing metal wind instruments: the rupture of the orbicularis muscle of the lips may severely impair a brass player's ability to play. A review of the literature and a description of the syndrome is executed, as well as an analysis of the various aspects of the disability assessment: damage produced, job evaluation, labor clinical judgment and evaluation of contingency.
\end{abstract}

Med Segur Trab (Internet) 2014; 60 (237) 779-785

Keywords: Orbicular, musician, Satchmo. 


\section{INTRODUCCIÓN}

Se trata de una patología relativamente frecuente en los músicos de viento pero escasamente conocida en la práctica clínica habitual. Por su propia naturaleza es potencialmente incapacitante y no parece haber muchas dudas de que tendría un origen profesional, pero dado que la población diana es poco numerosa seguramente es muy poco frecuente en expedientes de valoración de secuelas. Se presenta un caso promovido por una Mutua como Incapacidad Permanente (IP) derivada de accidente de trabajo (AT) y se hace una revisión de la literatura.

\section{RESUMEN DEL CASO}

Se trata de un paciente varón, de 35 años en la actualidad y con una doble actividad: trombón solista en una orquesta sinfónica y docente en dos empresas como profesor de trombón. Al parecer comienza con síntomas, molestias en los labios, dificultad para emitir las notas altas y fatigabilidad precoz a finales de 2009. Se le extiende parte de AT 24.08.2010 como solista de la orquesta con la siguiente descripción: «se le ha producido contractura en el labio". No queda claro si en esa fecha hubo un evento agudo en relación a su trabajo en la orquesta o un empeoramiento de algo previo, tampoco queda claro por qué el parte de AT se extiende en esa empresa concreta. Estuvo un periodo de baja de 24.8.10 a 2.8.11 por "otras alteraciones musculares específicas», al parecer el alta fue para pasar a la situación de excedencia en su puesto en la orquesta para dedicarse a la enseñanza. Fue tratado por múltiples especialistas tanto en España como en el extranjero y, especialmente, en el Instituto de Fisiología y Medicina del Arte de Tarrasa, curiosa institución que se dedica al tratamiento de las lesiones específicas de los artistas, especialmente los músicos.

El primer informe disponible de dicho instituto, llega al diagnóstico de «elongación del músculo orbicular de los labios» y recomienda reposo del instrumento, reacondicionamiento físico, electroestimulación e incrementos progresivos de la práctica musical. Sucesivos informes consignan mejoría, en marzo de 2011 se dice que ha llegado a tocar 120 minutos diarios. En el de julio de 2011 se dice que sigue con molestias, que percibe un cordón fibroso en el labio que por ecografía se corresponde a ligero engrosamiento contiguo al orbicular.

En cualquier caso fue alta y pasó a excedencia. Durante la misma tiene varias actividades, mayoritariamente de enseñanza. Se reincorpora a la orquesta el 1.9.13 y el 3.9.13 se extiende nuevo parte de AT e inicia nueva baja. El día 4.10 .13 se extiende alta por Informe Propuesta y su Mutua, propone IP Total.

Se dispone de nuevo informe del Instituto del Arte que dice que en la ecografía todavía se observa una cicatriz de $4 \mathrm{~mm}$ en el labio superior y que su situación es incompatible con su trabajo y que «desconocemos otros posibles tratamientos que puedan ser aplicados». Había sido visto por un cirujano Maxilofacial que prescribió férula de descarga por bruxismo. También fue visto por un eminente y mediático cirujano Plástico que desaconsejó la opción quirúrgica. Su Mutua entiende que el proceso está finalizado y que es imposible que mantenga la embocadura del trombón más de 1 hora y propone la IP, revisable en 18 meses pues dice que la patología es susceptible de mejoría con el reposo.

\section{DESCRIPCIÓN DEL SÍNDROME}

El síndrome fue descrito por primera vez en una comunicación por el Dr. Planas en $1982^{1}$. A pesar de que no es una condición infrecuente en los músicos que tocan instrumentos de viento de metal, hasta esa fecha no se había descrito el sustrato anatómico de la patología, ni se la había puesto nombre. El nombre "Satchmo", es el apodo que recibía el célebre trompetista de jazz, Louis Amstromg, del que se dice que en 1935 sufrió 
la lesión, lo que le obligó a dejar de tocar un tiempo y, por cierto, le indujo a empezar a cantar. A pesar de la relativa frecuencia de la patología hay pocas descripciones en la literatura, una búsqueda en PudMed o bases de datos similares de los términos: ruptura del orbicularis oris o Satchmo, apenas da 3-4 resultados. Probablemente porque afecta a un colectivo poco numeroso, especial diría yo, por lo que la falta de conocimiento de la patología es grande incluso en especialistas del área concreta: ORL, maxilofacial. De hecho el Dr. Planas es cirujano plástico.

Empezaré con un breve recuerdo anatómico funcional. Dentro de los instrumentos de viento, un subgrupo especial es el de instrumentos de metal. En estos, el sonido se produce después de que el músico adapte sus labios a la forma de la boquilla del instrumento herméticamente y haciendo vibrar los mismos dentro de la boquilla se produce un sonido que luego es amplificado por el propio instrumento. En otros, como los de madera, el sonido lo produce la vibración producida en una lengüeta que está en la boquilla al soplar por ella, pero en los de metal es la vibración de los labios (como si hiciéramos una "pedorreta", lo siento no encuentro una palabra alternativa) la que lo produce.

Este proceso de adaptación de la boca a la boquilla del instrumento es lo que se conoce como embocadura y es crítico en estos instrumentos. Afecta tanto a los labios, a la forma de los mismos, a la capacidad de contracción del orbicular, y a los dientes que están por detrás y producen el apoyo. La presión ejercida por un lado por la columna de aire generada contra los labios que sólo deben dejar de pasar una mínima cantidad, al tiempo que vibran y presionan con fuerza contra la boquilla metálica es muy importante, especialmente en las notas agudas, produciéndose incluso movilización de dientes, fisuras en los labios y, cómo no, elongaciones o roturas musculares. Y además, estas fuerzas combinadas no sólo deben acabar produciendo un sonido (eso quizás estaría al alcance de todos) sino que deben producir un sonido afinado de diferentes tonalidades y matices así como intensidades. La llave de todo el proceso (nunca mejor dicho) es un músculo del que se dice que su grosor en reposo es de $1 \mathrm{~mm}$ y de 3 contraído y que no tiene inserciones óseas.

Como en cualquier otro músculo que se rompe, la zona es reparada mediante una cicatriz, o sea un tejido no muscular y esto, además de molestias (el borde la boquilla, que algunos músicos describen como un cortador de galletas, se apoyaría sobre ella) produciría una pérdida del fino control que se debe tener sobre el músculo.

En la primera descripción del Dr. Planas se trataba de un músico trompetista profesional que a partir de un episodio agudo tenía dolor, fatigabilidad y dificultad para las notas altas. Su padre también trompetista había tenido que abandonar la profesión por síntomas similares. El Dr. Planas hace una exploración quirúrgica del labio, en este caso superior, y encuentra una rotura del orbicular cuyos extremos estaban unidos por material fibroso y las fibras posteriores estaban elongadas. Extirpó la zona fibrosa la unió con puntos y tras algún contratiempo con estos, el músico siguió tocando igual que antes. La publicación en forma de carta al director provocó una respuesta de un cirujano estadounidense que le felicita, pero, sobre todo, alaba su valor, pues hacer una incisión en el labio de un trompetista con tan pocos conocimientos previos y expectativas, él nunca se hubiera atrevido a hacerlo ${ }^{2}$.

El Dr. Planas publica una segundo caso en 1988, también operado y exitoso ${ }^{3}$. Esto produjo dos respuestas una de un músico profesional ${ }^{4}$ y otra del mismo cirujano estadounidense anterior.

En principio puede parecer sorprendente que se publique en una revista médica de Cirugía Plástica una respuesta de un músico profesional, no médico, sin embargo la más extensa y quizás principal publicación que se ocupa del síndrome son dos libros: Broken Embouchures y Embouchure Rehabilitation ${ }^{5}$ escritos por un músico, Lucinda Lewis, que incluso participa como invitada en simposios médicos sobre el tema y mantiene una página web www.embouchures.com. Además no sólo se ocupa de escribir sobre el tema sino que parece que diagnostica y pone pautas de tratamiento rehabilitador, así como 
proporciona adaptadores especiales para la embocadura. Es posible que sea la máxima autoridad mundial sobre la patología de la embocadura en músicos de viento.

Hay también una publicación de 1996 de una serie de 10 roturas del orbicular en músicos, operadas 9 con éxito ${ }^{6}$. Como se ve las publicaciones son, casi todas, relacionadas con la corrección quirúrgica del síndrome. A pesar de ello y teniendo en cuenta que hay poco publicado en revistas de tipo científico, parece que los especialistas específicos (incluida Lucinda Lewis), en publicaciones más ligeras, no consideran una buena solución la cirugía, pues extirpar una cicatriz produce otra quizás peor y se inclinan más por medidas más conservadoras (reposo durante meses, rehabilitación electroterapia, reeducación o cambio de la boquilla/embocadura). También se dice que si la situación del músico le aboca a dejar la carrera profesional se podría optar por cirugía como única salida ${ }^{7}$

No se mencionan en las publicaciones criterios diagnósticos más que la presentación de los síntomas en un músico de este tipo y dos posibles pruebas complementarias: electromiograma (EMG) para descartar una lesión nerviosa y la ecografía para intentar objetivar la rotura o el área cicatricial. Las fotos de los dos casos del Dr. Planas, antes y después de la cirugía, (y los artículos) están disponibles en la página web: www. clinicaplanas.com La simple contemplación de las fotos explica la dificultad para el diagnóstico para un médico que no conozca el tema (especialmente las fotos del «antes» sin ver el «después»).

\section{DISCUSIÓN Y VALORACIÓN}

Es un clásico en medicina evaluadora, el que cuando se quiere poner un ejemplo del balance entre limitaciones y requerimientos profesionales, de tal manera que menoscabos mínimos pueden dar lugar a una incapacidad por unos requerimiento del trabajo muy específicos, poner como ejemplo un músico (violinista, pianista) que pierde la falange distal de un dedo de la mano izquierda. Sin embargo este caso, que se ajusta casi al milímetro con el ejemplo clásico, es real. Como ejemplo además, permite reflexionar sobre muchos de los puntos conflictivos de cualquier evaluación, de alguna manera, creo que lleva el proceso de valoración a sus límites.

\section{DIAGNÓSTICO}

Respecto a la patología en sí, no es muy conocida, ni desde luego frecuente, como, por otra parte ocurre con muchas patologías específicas de los músicos profesionales. Como dije antes, yo no he encontrado una descripción de los requisitos necesarios para el diagnóstico más allá de los síntomas típicos. Respecto a las pruebas complementarias se citan el EMG para descartar una afectación neurológica y la ecografía identificaría la zona de ruptura (en el caso de que hubiera una rotura significativa, no me queda claro si una ecografía en ese músculo puede detectar una microrrotura o una elongación). Esto no es muy diferente a otras patologías musculares en actividades muy exigentes físicamente como las de los deportistas. Es una experiencia común en los que siguen la prensa deportiva, sobre todo el fútbol, leer que un futbolista tiene síntomas de ruptura fibrilar en una músculo largo y que tras ecografía o RM las encuentran (roturas o incluso microrroturas) y si no las encuentran se habla de una elongación, contractura, tirón, etc. Así parece haber un espectro de diferentes fases de la afectación desde una poco concreta como «la fatiga muscular de la embocadura» que se supone que es un sobre uso pero que sorprendentemente no siempre mejora con el reposo, a irritaciones locales, edemas, hematomas, fisuras, elongaciones del músculo y por fin roturas, que parece un todo continuo de menor a mayor sobre el mismo problema ${ }^{7}$. En cualquier caso el tratamiento es similar: reposo, medidas antiinflamatorias, correcciones de la técnica y de la boquilla y fisioterapia o terapia física con mejor pronóstico si no se identifica rotura. 
Las roturas fibrilares se reparan con una cicatriz, que no es tejido con la calidad del músculo y muchas roturas o repetidas o muy grandes pueden deteriorar mucho el músculo y limitar o impedir el uso intenso del mismo, por ejemplo en un deporte. Pues algo similar debe de ocurrir en el orbicular de los labios en músicos de viento, con las particularidad de que el músculo tiene unos centímetros y que más que potencia (que también) se le exige una precisión exquisita. En este caso agrava o dificulta el diagnóstico el que la lesión de este pequeño músculo no es frecuente en los medios clínicos habituales y requiere, incluso, de especialistas específicos.

\section{FRECUENCIA}

No conozco publicaciones específicas sobre la frecuencia del síndrome concreto. Lo publicado serían estimaciones generalmente derivadas de estudios epidemiológicos descriptivos sobre los síntomas más frecuentes en los músicos en general. Debido a la gran variedad de instrumentos y por tanto con condiciones ergonómicas absolutamente dispares, es difícil aislar una patología concreta. Se puede citar una artículo que hace una revisión de los problemas exclusivamente orofaciales en músicos, los más frecuentes serían «los problemas con la articulación témporo mandibular, las infecciones por virus del herpes, los problemas dentales y los problemas con la musculatura perioral ${ }^{8}$, aunque me parece que está sólo orientado a problemas de tipo estomatológico. Más interesante puede ser un estudio sobre los problemas de salud que afectan a los músicos hecho en Cataluña ${ }^{9}$ y que recoge un apartado concreto sobre músicos de viento, que junto con los percusionistas e instrumentos de cuerda frotada, son los que más frecuentemente tienen problemas. En concreto los instrumentistas de viento de metal, con el $85,5 \%$ son los segundos más frecuentes, cuando por otra parte no están entre los instrumentos más usados. En concreto los instrumentistas de viento metal que tuvieron problemas, en un $48,4 \%$ los referían a la boca. En general se suele citar en muchas publicaciones, científicas o no, que uno de cada 3 músicos de viento metal sufrirán problemas con la embocadura a lo largo de su vida. Una cosa sorprendente de este estudio en Cataluña es que la población estudiada eran músicos profesionales o no de esa Comunidad que llevaran más de dos años tocando y se enviaron nada menos que 9795 encuestas (contestaron 1730), aunque probablemente menos del 10\% eran músicos de viento y sólo el 33\% eran profesionales, pero igual el problema no es tan infrecuente.

\section{VALORACIÓN PROFESIONAL}

Este es un asunto controvertido o difícil de definir concretamente. Como ocurre en este caso los músicos profesionales pueden tener una vida laboral compleja con actividades simultáneas o con altas/bajas cortas y sucesivas. Por un lado puede estar súper especializado (solista en este caso), o menos con diferentes grados de exigencia (clásica, jazz, pop, bandas, etc.) o incluso dedicarse a la enseñanza. Cada una de estas circunstancias tiene unas peculiaridades bien distintas. En el caso concreto al tratarse de un AT, su profesión era a la que se dedicaba en el momento del AT y aunque en ese momento me constan 3 (solista, profesor y autónomo), es en la que se accidentó y se emitió el parte de AT (solista de orquesta) la profesión de referencia.

\section{JUICIO CLÍNICO LABORAL}

El artículo 136.1 de la Ley General de la Seguridad Social, define la IP contributiva como: En la modalidad contributiva, es incapacidad permanente la situación del trabajador que, después de haber estado sometido al tratamiento prescrito, presenta reducciones anatómicas o funcionales graves, susceptibles de determinación objetiva $y$ previsiblemente definitivas, que disminuyan o anulen su capacidad laboral. No obstará 
a tal calificación la posibilidad de recuperación de la capacidad laboral del inválido, si dicha posibilidad se estima médicamente como incierta o a largo plazo ${ }^{10}$. O sea el elemento fundamental de una IP de esta modalidad contributiva es por un lado hacer un balance entre la patología y los menoscabos que producen y por otro las exigencias del puesto de trabajo. Este juicio, este balance, se hace en el juicio clínico laboral.

En teoría y dependiendo de la graduación de la intensidad de los síntomas, el síndrome justifica limitaciones en cuanto a las horas en que se puede tocar de manera continua y especialmente en la capacidad de emitir algunos tonos o su intensidad, especialmente agudos. No se puede afirmar que la persona no pueda tocar el instrumento, sino que quizás no lo pueda tocar mucho tiempo o con una exigencia extrema. ¿Sería pues esto una disminución del rendimiento, más que una imposibilidad para hacer todas o las fundamentales tareas de la profesión?, por cierto ¿Cuáles son las tareas fundamentales de una profesión? También creo que hay que tener en cuenta la diferencia entre profesión genérica o puesto de trabajo concreto, aunque siempre me pareció que esto tiene escaso soporte legal, yo no conozco una definición de que es la profesión habitual. Un músico que toca el trombón de manera profesional, tanto lo puede hacer en una orquesta, como solista o no, en una grabación de un disco como músico de apoyo, en una banda municipal de música, en una orquesta que actúa en fiestas patronales, como dando clase de trombón. ¿Sería razonable pensar que alguien puede ser IP Total para solista de trombón en una orquesta sinfónica y compatibilizarlo con ser trombón no solista en un estudio de grabación o en una orquesta de música ligera? Sería razonable, aplicado al caso, ser IP Total para solista de trombón y compatibilizarlo, como ocurre, con dar clase o ser catedrático de trombón. Una última consideración sería que la ley General de la SS dice textualmente respecto a la IP en artículo 136: «reducciones anatómicas o funcionales graves". Aunque creo que pocas veces nos fijamos en esto, no creo que una rotura del orbicular de los labios se pueda considerar grave, ni siquiera funcionalmente.

\section{VALORACIÓN DE LA CONTINGENCIA}

Por último queda la consideración de la contingencia, creo que la causa de esta poco frecuente lesión es el trabajo y al no haber manera de incluirla como Enfermedad Profesional por no estar listada, la contingencia correcta, tal como hizo la Mutua, es la de AT, por el artículo 115, 2e: "Las enfermedades, no incluidas en el artículo siguiente, que contraiga el trabajador con motivo de la realización de su trabajo, siempre que se pruebe que la enfermedad tuvo por causa exclusiva la ejecución del mismo». Por lo publicado la frecuencia de dolencias que pueden tener alguna relación con el trabajo en músico profesionales es muy alta, la mayoría serían patologías del aparato locomotor que en muchos casos se podrían encajar bien en la vigente lista de EP, caso de las tendinitis. No así en el caso de la patología de espalda que creo tendría mal encaje incluso como AT o una peculiar patología que parece es la que más preocupa en el sector: la distonía (con similitudes al "calambre del escribiente») que parece a caballo entre la neurología y la psiquiatría.

\section{REFERENCIAS BIBLIOGRÁFICAS}

1. Rupture of the Orbicularis Oris in Trumpet Players (Satchmo's Syndrome). Jaime Planas, M.D. April 1982, Plastic and Reconstructive Surgery. April 1982.

2. Rupture of the Orbicularis Oris in Trumpet Players (Satchmo's Syndrome) Discussion. Bernard I., Kaye M.D., D.M.D. April 1982, Plastic and Reconstructive Surgery

3. Further experience with Rupture of the Orbicularis Oris in Trumpet Players. Jaime Planas, M.D. June 1988, Plastic and Reconstructive Surgery.

4. Further Experience with Rupture of the Orbicularis Oris in Trumpet Players. Discussion. Philip Farkas, D. Mus. June 1988, Plastic and Reconstructive Surgery.

5. Lewis, Lucinda. Broken Embouchures. Broken Embouchures. s.1.: Embouchures.com, Inc., 2005. 
6. Orbicularis oris muscle injury in brassplayers. Papsin BC, Maaske LA, McGrail JS. 1996 Jun, Laryngoscope., págs. 106(6):757-60.

7. Problemas embocadura I-VI. Llobet, Jaume Rosset i. 2005, 12 Notas (disponible en http://www.institutart. $\mathrm{com} /)$.

8. Problemas orofaciales en músicos: una revisión de la literatura. Rodríguez-Lozano FJ, Saéz-Yuguero MR y otros. 2011, Med Probl Perform Art.

9. Detección de factores de riesgo en los músicos de Cataluña. Roset-Llobet, Jaume, Rosinés-Cubells, Dolors y Saló-Orfila, Josep M. 2000, Medl Probl Perform Art, págs. 15: 167-174.

10. Real Decreto Legislativo 1/1994, de 20 de junio, por el que se aprueba el Texto Refundido de la Ley General de la Seguridad Social. Ley General de la Seguridad Social.

|| 